\title{
Off-line robustification of Generalized Predictive Control for uncertain systems
}

\author{
KHELIFA KHELIFI OTMANE, NORDINE BALI and LAZHARI NEZLI
}

\begin{abstract}
An off-line methodology was proposed for enhancing the robustness of an initial Generalized Predictive Control (GPC) by convex optimization of the Youla parameter. However, this procedure of robustification is restricted with the case of the systems affected only by unstructured uncertainties. This paper proposes an extension of this method to the systems subjected to both unstructured and structured polytopic uncertainties. The main idea consists in adding supplementary constraints to the optimization problem which validates the Lipatov stability condition at each vertex of the polytope. These polytopic uncertainties impose a set of non convex quadratic constraints. The globally optimal solution is found by means of the GloptiPoly3 software. Therefore, this robustification provides stability robustness towards unstructured uncertainties for the nominal system, while guaranteeing stability properties over a specified polytopic domain of uncertainties. Finally, an illustrative example is given.
\end{abstract}

Key words: Generalized Predictive Control, polytopic uncertainties, relaxation, robust control, Youla parametrization

\section{Introduction}

Model Predictive Control (MPC) is an advanced method for systems control that has been used in several process industries like chemical plants, oil refineries and in area of robotics. When focusing on linear discrete time transfer function models and quadratic cost functions, some of the best known approaches include the Generalized Predictive Control (GPC) [3], its related algorithms with guaranteed nominal stability as presented e.g. in [4], the inner loop stabilizing Stable Predictive Control [5] and the Cautious Stable Predictive Control [6]. The presence of the plant model is a necessary element for the development of the predictive control. Therefore the success of GPC depends on the degree of precision of the plant model. Generally, there exist two typical descriptions of model uncertainties, depending on how these uncertainties are described, structured (parametric) or unstructured uncertainties.

K.K. Otmane and L. Nezli are with the Process Control Laboratory, Ecole Nationale Polytechnique, ENP, Algers, e-mails: khelifi_ot_uhbc@yahoo.fr, 1_nezli@yahoo.fr. N. Bali is with the Industrial Systems Laboratory, University of Sciences and Technology Houari Boumediene, Algiers, e-mail: nbali@usthb.fr.

Received 19.03.2014. 
The GPC robustness issue in the presence of unstructured modeling errors is usually dealt with by enhancing the robustness of existing designs by introducing degrees of freedom based on the Youla parametrization, also called as $Q$-parametrization. This parametrization used in [5], [7], [8] and [9] where a robust optimization problem is defined and the Youla parameter $Q$ is derived. However, these methods present some shortcomings. For example, the method proposed in [5] provides high robustness bounds but penalizes the disturbance rejection performance. Also, the methods developed in [7] and [8] defined a mixed sensitivity problem, but the trade-off between robustness and nominal performance is difficult to adjust. This difficulty is surmounted by the method proposed in [9] but the Youla parameter is searched for in a very restricted space.

Another sophisticated methodology has been developed recently in [1] to enhance the robustness of the GPC controller towards unstructured uncertainties while respecting time-domain constraints. This methodology starts with the design of an initial stabilizing GPC controller; this controller is then robustified via the Youla parametrization which permits to access all the stabilizing controllers. This parametrization allows formulating frequency and time-domain constraints as convex optimization.

The last approach of robustification constitutes a paramount advantage for a good regulation of a process subjected to unstructured uncertainties. This paper presents an extension of this methodology to the systems subjected to both unstructured and polytopic structured uncertainties, while preserving the same formalism by Youla parametrization adopted in [1].

This paper is organized as follows. Section 2 reminds the main steps leading to the design of an initial GPC controller. Section 3 considers Youla parametrization of the system with the initial controller. The robustification procedure, based on the Youla parameter, under unstructured uncertainties is reminded in Section 4. Section 5 provides the main result, the elaboration of a robustified controller under both unstructured and polytopic uncertainties. This control strategy is applied in Section 6 to the speed control of an induction machine. Finally, some concluding remarks are presented in Section 7.

\section{Design of the GPC law}

The GPC control law uses the controlled auto-regressive integrated moving average (CARIMA) model:

$$
A\left(q^{-1}\right) y(t)=B\left(q^{-1}\right) u(t)+\frac{\xi(t)}{\Delta\left(q^{-1}\right)}
$$

where $u(t)$ and $y(t)$ are the plant input and output for a SISO system. $A$ and $B$ are polynomials in backward shift operator $q^{-1}$ and $\Delta\left(q^{-1)}\right)=1-q^{-1}$. $\xi(t)$ is an uncorrelated random sequence. The $j$-step ahead prediction over the costing horizons $N_{1} \leqslant j \leqslant N_{2}$ is given by: 


$$
\begin{aligned}
y(t+j) & =\underbrace{F_{j}\left(q^{-1}\right) y(t)+H_{j}\left(q^{-1}\right) \Delta u(t-1)}_{\text {freeresponse }} \\
& +\underbrace{G_{j}\left(q^{-1}\right) \Delta u(t+j-1)+J_{j}\left(q^{-1}\right) \xi(t+j)}_{\text {forcedresponse }} .
\end{aligned}
$$

$F_{j}, G_{j}, H_{j}$ are polynomials obtained by solving Diophantine equations. To achieve optimal command values, the GPC uses a quadratic cost function defined as:

$$
J\left(N_{1}, N_{2}\right)=\sum_{j=N_{1}}^{N_{2}}[\hat{y}(t+j)-w(t+j)]^{2}+\lambda \sum_{j=1}^{N_{u}} \Delta[u(t+j-1)]^{2}
$$

where $\Delta u(t+j)=0$ for $j \geqslant N_{u} . N_{1}$ and $N_{2}$ define the output prediction horizons, and $N_{u}$ the control horizon. $\lambda$ is the control weighting factor, $w$ is the reference value, $\hat{y}$ is the predicted output value. The receding horizon principle assumes that only the first value of the optimal control sequence resulting from the minimization of (3) is applied, so that at the next sampling period the same procedure is repeated. This control strategy leads to a 2-DOF RST controller implemented through a difference equation:

$$
S\left(q^{-1}\right) \Delta\left(q^{-1}\right) u(t)=-R\left(q^{-1}\right) y(t)+T(q) w(t)
$$

Assuming the design has been performed with $R_{0}, S_{0}, T_{0}$ and $N_{1}, N_{2}, N_{u}, \lambda$ adjusted to satisfy certain closed loop performance. The resulting 2-DOF $R S T$ controller will be denoted $R_{0}, S_{0}, T_{0}$ (Fig. 1).

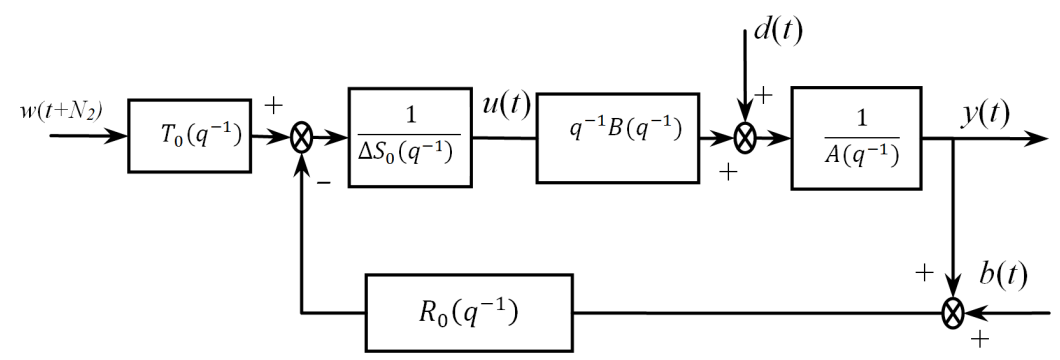

Figure 1: GPC Equivalent polynomial RST controller. 


\section{Youla parametrization}

As given in [1], the Youla parametrization of the previous initial Generalized Predictive Controller $\left(R_{0}, S_{0}, T_{0}\right)$ leads to the following stabilizing polynomials:

$$
\left\{\begin{array}{c}
T\left(q^{-1}\right)=T_{0}\left(q^{-1}\right)-A_{0}\left(q^{-1}\right) Q_{2}\left(q^{-1}\right) \\
R\left(q^{-1}\right)=R_{0}\left(q^{-1}\right)+\Delta A\left(q^{-1}\right) Q_{1}\left(q^{-1}\right) \\
S\left(q^{-1}\right)=S_{0}\left(q^{-1}\right)-q^{-1} B\left(q^{-1}\right) Q_{1}\left(q^{-1}\right)
\end{array}\right.
$$

where $Q_{1}$ and $Q_{2}$ are free stable transfer functions. And $P_{0}=A_{0} A_{c}=\Delta A S_{0}+q^{-1} B R_{0}$ is the characteristic polynomial of the closed loop obtained with the initial controller where $A_{c}$ and $A_{0}$ represent, respectively, a control polynomial and an observer polynomial.

The corresponding block diagram of the controller (5) is shown in Fig. 2. So, two remarks can be done: $Q_{2}$ modifies only the tracking behavior, and, if the model is exact, the characteristic equation is not modified by the parametrization:

$$
\Delta A S_{0}+q^{-1} B R_{0}=\Delta A S+q^{-1} B R
$$

whereas parameter $Q_{1}$ modifies the closed loop features keeping the input-output transfer unchanged.

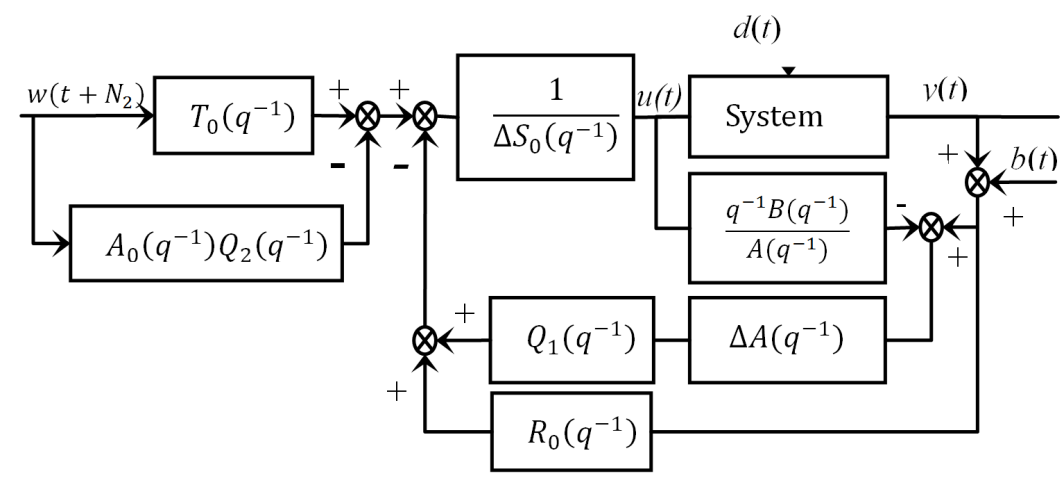

Figure 2: RST controller with Youla parametrization.

In the next developments, $Q_{2}$ will be set to zero assuming that the tracking performance is ensured by the initial GPC controller design and $Q_{1}$ will be used to robustify this initial controller. As a result, the closed-loop transfer between $w$ and y becomes

$$
\frac{y}{w}=\frac{T_{0} q^{-1} B}{P_{0}}
$$




\section{Robust stability under unstructured uncertainties}

Without loss of generality, and for simplification purposes, the particular case of the maximization of the robust stability under additive direct unstructured uncertainties is considered below. This uncertainty is shown in Fig. 3. $P\left(q^{-1}\right)$ represents the system connected to the uncertainty block.

$$
P=\frac{v}{z}=-\frac{R_{0} A}{P_{0}}-\frac{A^{2} \Delta}{P_{0}} Q_{1}
$$

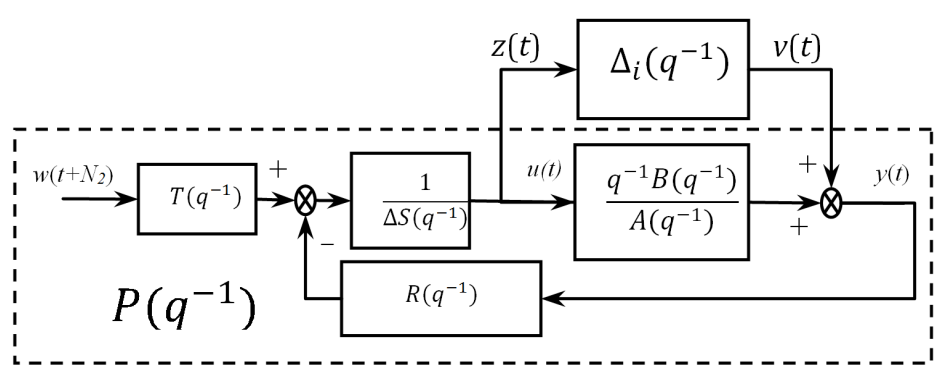

Figure 3: Unstructured additive direct uncertainty.

According to the small gain theorem [9], robustness under unstructured uncertainties is maximized formulating a $H_{\infty}$ norm minimization:

$$
\min _{Q_{1} \in \mathcal{R} H_{\infty}} P\left(q^{-1}\right) W\left(q^{-1}\right)_{\infty}
$$

where the weighting $W$ reflects the frequency range in which model uncertainties are more significant, and $\mathcal{R} H_{\infty}$ is the space of all proper and stable transfer functions. The Youla parametrization allows linear dependency between $P$ and the Youla parameter $Q_{1}$ as shown in equation (8). So, the specifications defined by equation (9) are convex in $Q_{1}$. This convex optimization problem leads to a $Q_{1}$ parameter varying in an infinite dimensional space [1]. To the author's knowledge, there is no solution to this optimization problem, and a sub-optimal solution can be obtained by considering a finite dimensional sub-space generated by an orthonormal base of discrete stable transfer functions such as a polynomial or FIR (Finite Impulse Response) filter:

$$
Q_{1}=\sum_{i=0}^{n_{q}} \alpha_{i} Q_{1 i}
$$

Then, the $H_{\infty}$ norm minimization can be approximated by a minimization under linear inequality constraints, such as the equation (9) can be written as

$$
\min _{Q_{1} \in \mathcal{R} \mathrm{H}_{\infty}} T_{1}+T_{2} Q_{1 \infty}=\min _{Q_{1} \mathcal{R} H_{\infty} w, 0 \leqslant w \leqslant \pi} \max _{\mid}\left|T_{1}\left(e^{-j w}\right)+T_{2}\left(e^{-j w}\right) Q_{1}\left(e^{-j w}\right)\right|
$$


with the definition of $Q_{1}$ given in (9), and replacing half of the unit disk by a finite grid, this relation becomes

$$
|\underbrace{T_{1}\left(e^{-\theta_{k}}\right)}_{T_{1 k}}+\underbrace{T_{2}\left(e^{-\theta_{k}}\right)\left[\begin{array}{lll}
Q_{10}\left(e^{-\theta_{k}}\right) & \ldots & Q_{1 n_{q}}\left(e^{-\theta_{k}}\right)
\end{array}\right]}_{\mathbf{T}_{2 \mathbf{k}}} \times \underbrace{\left[\begin{array}{c}
\alpha_{0} \\
\vdots \\
\alpha_{n_{q}}
\end{array}\right]}_{\alpha}| \leqslant \gamma
$$

with $\gamma$ which is the upper bound of the left-hand side of the equation (11), and $\theta_{k}=$ $\pi(k-1) /(N-1)$ for $k=1, \ldots, N$, leading to the relation

$$
\left|T_{1 k}+\mathbf{T}_{2 k}\right| \leqslant \gamma \text { for } k=1, \ldots, N .
$$

This matrix inequality under the form $|u| \leqslant \gamma$, is approximated by a set of four linear inequalities

$$
\begin{array}{rr}
\operatorname{Re}(u)+\operatorname{Im}(u) \leqslant \gamma, \quad \operatorname{Re}(u)-\operatorname{Im}(u) \leqslant \gamma \\
-\operatorname{Re}(u)+\operatorname{Im}(u) \leqslant \gamma, \quad-\operatorname{Re}(u)-\operatorname{Im}(u) \leqslant \gamma
\end{array}
$$

As given in [1], these inequalities lead to the cost minimization under inequality constraints. Hence, the stability robustness problem towards additive unstructured uncertainties can be written as follows

$$
\min _{\mathbf{A X}-\mathbf{B} \leqslant 0} \mathbf{C X}
$$

with

$$
\begin{aligned}
& \mathbf{A}=\left[\begin{array}{cc}
-1 & \operatorname{Re}\left(\mathbf{T}_{21}\right)+\operatorname{Im}\left(\mathbf{T}_{21}\right) \\
\vdots & \vdots \\
-1 & -\operatorname{Re}\left(\mathbf{T}_{2 N}\right)-\operatorname{Im}\left(\mathbf{T}_{2 N}\right) \\
-1 & 0 \cdots \cdots \cdots 0
\end{array}\right]_{(4 N+1) \times\left(n_{q}+2\right)} \\
& \mathbf{B}=\left[\begin{array}{c}
-\operatorname{Re}\left(T_{11}\right)-\operatorname{Im}\left(T_{11}\right) \\
\vdots \\
-\operatorname{Re}\left(T_{1 N}\right)-\operatorname{Im}\left(T_{1 N}\right) \\
0
\end{array}\right]_{(4 N+1) \times 1}\left[\begin{array}{c}
\gamma \\
\alpha_{0} \\
\cdots \\
\alpha_{n_{q}}
\end{array}\right]_{\left(n_{q}+2\right) \times 1}, \mathbf{X}=\left[\begin{array}{ccc}
1 & \cdots & 0
\end{array}\right]_{1 \times\left(n_{q}+2\right)}
\end{aligned}
$$




\section{Robust stability under polytopic uncertainties}

This section formulates the main result of the paper which consists into guaranteeing the robust stability under polytopic uncertainties. The procedure is the following: firstly an initial stabilizing controller is designed for the nominal plant, which is then robustified under unstructured uncertainties based on the $Q_{1}$ parameter as given in section 4. Since the initial stabilizing controller or the robustified nominal controller can be unstable for some regions of the polytopic uncertain domain, a suitable method that guarantees stability on the entire polytopic domain is further investigated.

Consider a polytopic system with $l$ vertices such that the $i$ th vertex constitutes the transfer function of a model $G_{i}$ such that:

$$
G_{i}\left(q^{-1}\right)=q^{-1} \frac{B_{i}\left(q^{-1}\right)}{A_{i}\left(q^{-1}\right)}
$$

Thus, the whole polytope (Fig. 4) can be denoted by:

$$
\Omega=\operatorname{Co}\left\{\left[\begin{array}{ll}
A_{1} & B_{1}
\end{array}\right],\left[\begin{array}{ll}
A_{2} & B_{2}
\end{array}\right], \cdots,\left[\begin{array}{ll}
A_{l} & B_{l}
\end{array}\right]\right\}
$$

with 'Co' standing for the convex hull defined by $l$ vertices $\left[A_{i} B_{i}\right], i=1, \ldots, l$.

As $\Omega$ is a polytope, thus a convex set, guaranteeing stability of (16) for the entire space $\Omega$ is equivalent to guarantee the stability for all the vertices of the uncertain polytopic domain [10].

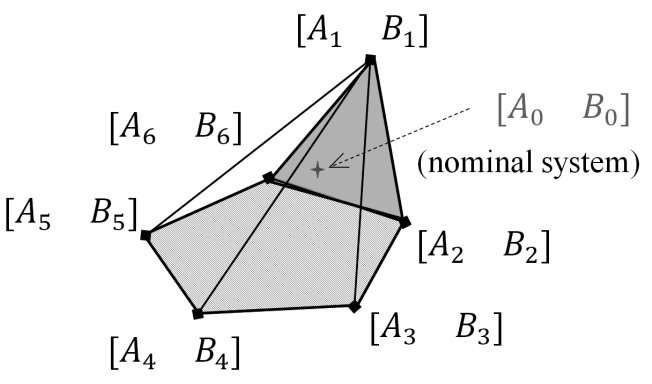

Figure 4: Polytopic uncertainty representation with $l=6$.

In this multi-model case, the closed-loop transfer from the input $w$ to the output $y$ (Fig. 2) is derived as

$$
\frac{y}{w}=\frac{T_{0} q^{-1} B_{i}}{P_{i}}
$$

where $P_{i}$ represents the closed loop characteristic polynomial of the $i$ th vertex of the domain $\Omega$ given by

$$
P_{i}=\Delta A_{i} S_{0}+q^{-1} B_{i} R_{0}+q^{-1} \Delta Q_{1}\left(B_{i} A-A_{i} B\right)
$$


Hence, the problem of checking the stability of the polytopic system reduces to the determination of whether or not the roots of each characteristic polynomial $P_{i}$ lie inside the unit circle or not. At this step, the difficulty appears due to the presence of $Q_{1}$ parameter in (19). It can be noticed easily that if the model is exact (i.e. if $A=A_{i}$ and $B=B_{i}$ ), then the closed-loop transfer (18) is reduced to (7). So, let us rewrite the expression (19) as

$$
P_{i}=\widetilde{T}_{1 i}+\widetilde{T}_{2 i} Q_{1} .
$$

Also, we can write

$$
P_{i}=\sum_{j=0}^{n} p_{i j} q^{-j}, \quad \widetilde{T}_{1 i}=\sum_{j=0}^{n_{1}} t_{1 i j} q^{-j}, \quad \text { and } \quad \widetilde{T}_{2 i}=\sum_{j=0}^{n_{2}} t_{2 i j} q^{-j}
$$

with out loss of generality, let us assume that $n_{2}+n_{q} \geqslant n_{1}$. This means that $n=n_{2}+n_{q}$.

Now, for $Q_{1}$ expressed as in (10), it is possible to formulate the characteristic polynomial coefficients $p_{i j}$ in terms of $\alpha_{i}$ parameters. This relation is given in matrix form as

$$
\boldsymbol{P}_{i}=\boldsymbol{L}_{i}+\boldsymbol{H}_{i} \boldsymbol{\alpha}
$$

with

$$
\begin{aligned}
& \boldsymbol{P}_{i}=\left[\begin{array}{c}
p_{i 0} \\
p_{i 1} \\
\vdots \\
p_{\text {in }}
\end{array}\right]_{(n+1) \times 1}, \quad \boldsymbol{L}_{i}=\left[\begin{array}{c}
t_{1 i 0} \\
\vdots \\
t_{1 \text { lin }_{1}} \\
0 \\
\vdots \\
0
\end{array}\right]_{(n+1) \times 1} \\
& \boldsymbol{H}_{i}=\left[\begin{array}{cccc}
t_{2 i 0} & 0 & \ldots & 0 \\
t_{2 i 1} & t_{2 i 0} & \ldots & 0 \\
\vdots & t_{2 i 1} & \ldots & \vdots \\
t_{2 i n_{2}} & \vdots & \ldots & t_{2 i 0} \\
0 & t_{2 i n_{2}} & \ldots & t_{2 i 1} \\
0 & 0 & \ldots & \vdots \\
\vdots & \vdots & \ldots & \vdots \\
0 & 0 & \ldots & t_{2 i n_{3}}
\end{array}\right] \\
& \boldsymbol{\alpha}=\left[\begin{array}{c}
\alpha_{0} \\
\alpha_{1} \\
\vdots \\
\alpha_{n_{q}}
\end{array}\right]_{\left(n_{q}+1\right) \times 1}, \text { and } n_{3}=\left\{\begin{array}{lll}
n_{q}-n_{2} & \text { if } & n_{2}<n_{q} \\
n_{q} & \text { if } & n_{2} \geqslant n_{q}
\end{array}\right.
\end{aligned}
$$




\section{Stability condition}

In 1978, Lipatov and Sokolov [11] developed the improved sufficient conditions for stability and instability of continuous linear stationary systems through Hurwitz stability and instability conditions. It turns out that the results are in terms of coefficients of characteristic polynomials of the systems. In the following, we state a result of theirs.

Let

$$
F(s)=\sum_{k=0}^{n} a_{k} s^{k}, a_{k}>0 \text { for } k=0,1, \ldots, n
$$

be a polynomial with real coefficients. A sufficient condition for the above polynomial to be Hurwitz stable (roots in the open left-half plane) is [11]

$$
a_{j-1} a_{j+2} \leqslant 0.4655 a_{j} a_{j+1}, \quad j=0,1, \ldots, n-2 .
$$

In order to apply the above condition to our procedure, the characteristic polynomial (21) must be converted to the continuous form. For that purpose, the bilinear transform is used. The bilinear transform is also known as Tustin's rule as well as the more familiar Trapezoidal rule used in numerical integration [12]. Here, the backward shift operator $q^{-1}$ is replaced by

$$
q^{-1}=\frac{1-(T / 2) s}{1+(T / 2) s}
$$

where $T$ is the sample time. Then, the equivalent characteristic polynomial in continuous time is obtained. It is denoted by asterisk.

$$
P_{i}^{*}(s)=\left(1+\frac{T}{2} s\right)^{n} P_{i}\left(\frac{1-(T / 2) s}{1+(T / 2) s}\right)=\sum_{j=0}^{n} p_{i j}^{*} s^{j}
$$

The relationship between the elements $p_{i j}$ and $p_{i j}^{*}$ is given by the following matrix equation [13], [14]:

$$
\boldsymbol{P}_{i}^{*}=\boldsymbol{Q}_{n} \boldsymbol{P}_{i}
$$

The transformation matrix $\boldsymbol{Q}_{n}$ is derived as follows:

$$
\boldsymbol{Q}_{n}=\left[\begin{array}{ccc}
(T / 2)^{0} q_{0,0} & \ldots & (T / 2)^{0} q_{0, n} \\
\vdots & \ddots & \vdots \\
(T / 2)^{n} q_{n, 0} & \ldots & (T / 2)^{n} q_{n, n}
\end{array}\right]
$$

with

$$
\begin{array}{ll}
q_{0, j}=1, & j=0,1, \ldots, n \\
q_{i, 0}=n ! /[(n-i) ! i !], & i=0,1, \ldots, n \\
q_{i, j}=q_{i, j-1}-q_{i-1, j-1}-q_{i-1, j}, & i, j=1, \ldots, n .
\end{array}
$$


From (21), the relation (26) becomes

$$
\boldsymbol{P}_{i}^{*}=\boldsymbol{L}_{i}^{*}+\boldsymbol{H}_{i}^{*} \boldsymbol{\alpha}
$$

such that

$$
\boldsymbol{L}_{i}^{*}=\boldsymbol{Q}_{n} \boldsymbol{L}_{i} \text { and } \boldsymbol{H}_{i}^{*}=\boldsymbol{Q}_{n} \boldsymbol{H}_{i} .
$$

Hence, after some developments, the Lipatov stability condition (23) has been transformed into the following quadratic constraints, generally, this set of constraints is non convex

$$
\boldsymbol{\alpha}^{T} \boldsymbol{W}_{i j} \boldsymbol{\alpha}+\boldsymbol{V}_{i j}^{T} \boldsymbol{\alpha}+\boldsymbol{U}_{i j} \leqslant 0 \text { for } j=1, \ldots,(n-2)
$$

where, for $j=1, \ldots,(n-2), \boldsymbol{W}_{i j}$ is known real symmetric $\left(n_{q}+1\right) \times\left(n_{q}+1\right)$ matrix, $\boldsymbol{V}_{i j}$ is known real $\left(n_{q}+1\right)$ vector, and $\boldsymbol{U}_{i j}$ is known real scalar. Therefore, guaranteeing the stability for all the vertices of the uncertain polytopic domain is equivalent to satisfying the $(n-2)$ inequalities defined by (29) for each vertex $i$ of the domain, it means $(n-$ 2) $l$ inequalities for all vertices. In conclusion, guaranteeing robust stability under both unstructured and polytopic uncertainties is globally achieved by adding the constraints (29) to the optimization problem (15).

In order to find the optimal solution of this problem, the latest version of the global polynomial optimization software GloptiPoly 3 developed in [2] has been used. Indeed, with the help of the solver SeDuMi [16], GloptiPoly 3 builds and solves a hierarchy of successive semidefinite programming (SDP), or convex linear matrix inequality (LMI) relaxations of increasing order, whose optima are guaranteed to converge monotonically to the global optimum. The main feature of this solver is that it can transcend the difficulties normally associated with non-convex optimization. Moreover, the optimal solution is reached at a relatively low computational cost.

\section{Example}

This part focuses on the results obtained while applying the previous robustification methodology to the velocity control of an induction machine. Starting from the identified transfer function between the torque $\Gamma_{m}$ and the velocity $\Omega_{m}$ for a sampling period $T=$ 5ms:

$$
\frac{\Omega_{m}}{\Gamma_{m}}=\frac{a q^{-1}+b q^{-2}}{1+c q^{-1}+d q^{-2}}
$$

with

$$
a=1.344, \quad b=3.024, \quad c=-0.98, \text { and } d=-0.02 .
$$

With this model, an initial GPC controller (called GPC0) has been first designed for $C\left(q^{-1}\right)=1$, and the following tuning parameters, selected according to the rules given in [17], $N_{1}=1, N_{2}=8$ and $\lambda=200$. Afterwards, it is considered that the nominal system 
is affected by high frequency neglected dynamics, represented as additive unstructured uncertainties. Thus, the following weighting $W\left(q^{-1}\right)$ is considered:

$$
W\left(q^{-1}\right)=\frac{1-0.8 q^{-1}}{0.2}
$$

Solving the optimization problem (15) provides a Youla parameter as a polynomial of a chosen order $n_{q}=6$. Fig. 5 shows measurement noise/control transfer function for the nominal system before robustification (GPC0) and after robustification under additive unstructured uncertainties (RGPC0). It can be noticed that the $H_{\infty}$ norm has been reduced using RGPC0. Therefore the robust stability for the nominal system with respect to highfrequency additive unstructured uncertainties is improved.

The next part refers to the robustification under polytopic uncertainties. Let us consider that the parameters $a, b, c$ and $d$ in (30), are uncertain up to $\pm 30 \%$ of their nominal values. This leads to a four-dimensional hypercube with $2^{4}=16$ vertices.

From Fig. 6 and 7, it can be noticed that the closed-loop of the polytopic uncertain system with both the initial controller GPC0 and RGPC0 are unstable for some vertices. Also we noticed that the RGPC0 destabilizes some vertices, which are initially stable with the GPC0. This difficulty justifies the design of other robustified controllers that will guarantee the stability under polytopic uncertainties. For that purpose, the robustified controller RGPC1 is designed by adding a set of quadratic constraints defined by (29) to the optimization problem. To solve this problem, we applied GloptiPoly 3 [2] solver. In order to obtain an accurate solution, we chose a relaxation order equal to 2 and we let $\mathrm{SeDuMi}$ minimize the duality gap as much as possible. The optimal value $\gamma=0.1685$ is obtained with the following $Q_{1}$ parameter:

$$
\begin{gathered}
Q_{1}\left(q^{-1}\right)=-0.0543-0.0214 q^{-1}-0.0052 q^{-2}+0.0042 q^{-3}+0.0087 q^{-4} \\
+0.0102 q^{-5}+0.0139 q^{-6}
\end{gathered}
$$

The closed-loop poles of all the vertices of the polytopic system with RGPC1 are shown in Fig. 8. As can be observed, the controlled system is stable for the entire polytopic domain. But the robust stability for the nominal system is decreased in comparison with the result obtained with RGPC0 for the nominal system (Fig. 5). It can be easily stated that a compromise has been achieved: with RGPC1 the robustness under unstructured uncertainties is less improved compared to RGPC0, but the robustness under polytopic uncertainties is satisfied. On the other hand, we noticed that with an increase of the degree of the Youla parameter, performances can still be improved to the detriment of the computational time.

\section{Conclusion}

This paper has presented an extending off-line methodology which enables robustifying an initial GPC controller using Youla parametrization and Lipatov stability crite- 


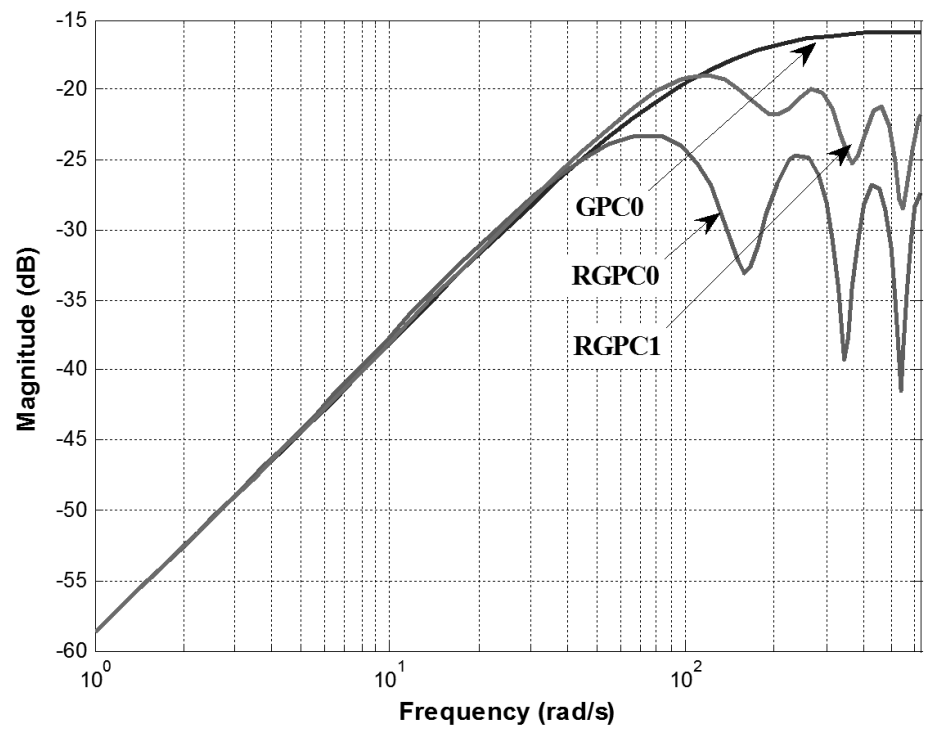

Figure 5: Measurement noise/control (with the nominal system) before and after robustification.

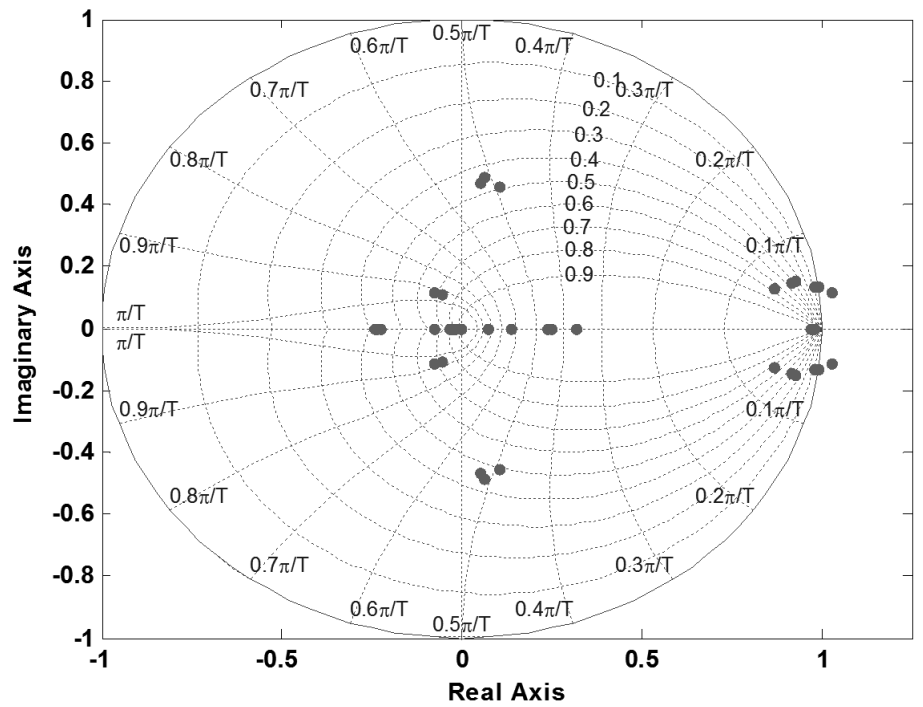

Figure 6: Closed-loop poles of all vertices of the polytopic system with the GPC0 controller.

rion. The major advantage consists in managing the compromise between robust stability under unstructured uncertainties for a nominal system and the robust stability under 


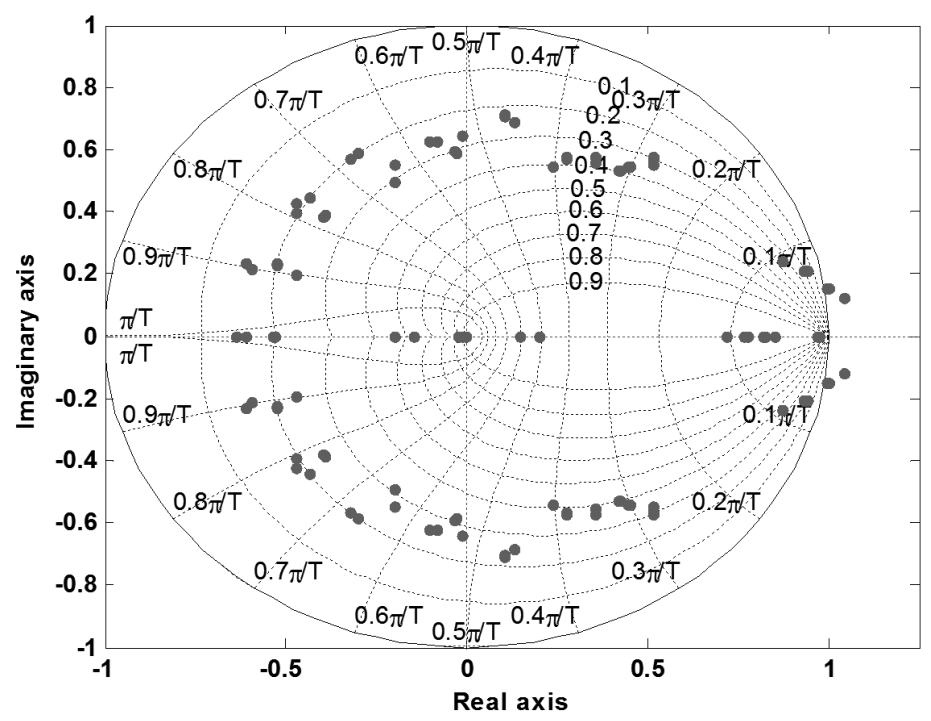

Figure 7: Closed-loop poles of all vertices of the polytopic system with the RGPC0 controller.

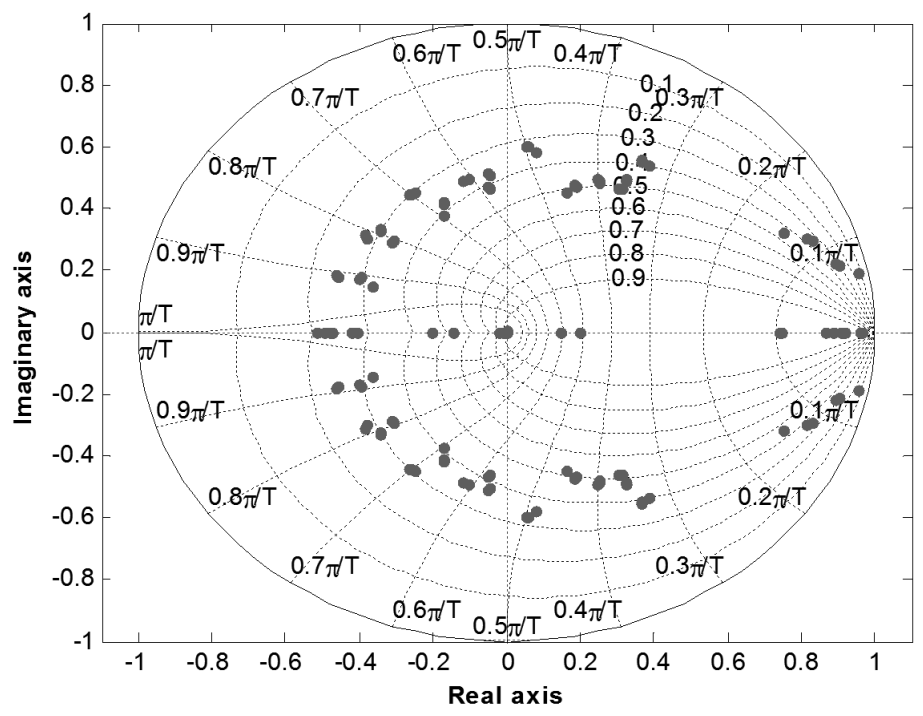

Figure 8: Closed-loop poles of all vertices of the polytopic system with the RGPC1 controller.

polytopic uncertainties for an entire variation domain, leading to an additional set of non convex quadratic constraints. An existing solver GloptiPoly 3 has been used to solve this optimization problem. 
The main contribution of this work consists in imposing the Lipatov stability condition to stabilize the controlled system for the entire polytopic domain, giving robustness properties, even if the system coupled with the initial controller is unstable in some points of the polytopic domain. This means that the proposed method offers a way to increase the polytopic domain for which the stability is guaranteed. Indeed, this robustification method can be also applied to any RST controller, not necessarily predictive.

\section{References}

[1] P. Rodriguez and D. Dumur: Generalized Predictive Control robustification under frequency and time-domain constraints. IEEE Trans. on Control Systems Technology, 13(4), (2005), 577-587.

[2] D. Henrion, J.B. Lasserre and J. Löfberg: GloptiPoly 3: Moments, optimization and semidefinite programming. Optimization Methods and Software, 24(4), (2009), 761-779.

[3] D.W. Clarke, C. Mohtadi and P.S. Tuffs: Generalized Predictive Control. Part 1 and 2. Automatica, 23(2), (1987), 137-160.

[4] H. Demircioglu and D.W. Clarke: Generalised Predictive Control with endpoint state weighting. IEE Proceedings, Part D, 140(4), (1993), 275-282.

[5] B. Kouvaritakis, J.A. Rossiter and A.O.T. Chang: Stable Generalised Predictive Control: An algorithm with guaranteed stability. IEE Proceedings, Part D, 139(4), (1992), 349-362.

[6] J.R. Gossner, B. Kouvaritakis and J.A. Rossiter: Cautious stable predictive control: A guaranteed stable predictive control algorithm with low input activity and good robustness. Int. l J. of Control, 67(5), (1997), 675-697.

[7] K. Hrissagis, O.D. Crisalle and M. Sznaier: Robust design of unconstrained predictive controllers. American Control Conf., Seattle, Washington, (1995).

[8] R.A.J. De VRIES and T.J.J. VAN DEN Boom: Constrained robust predictive control. European Control Conf., Rome, Italy, (1995).

[9] M. Morari and E. Zafiriou: Robust Process Control. Prentice Hall, Englewood Cliffs, N.J. 1989.

[10] M.V. Kothare, V. Balakrishnan and M. Morari: Robust constrained model predictive control using linear matrix in-equalities. Automatica, 32(10), (1996), 1361-1365. 
[11] A.V. LipATOV and N.I. SOKOLOV: Some sufficient conditions for stability and instability of continuous linear stationary systems. Automation and Remote Control, 39(9), (1978), 1285-1291.

[12] K. Ogata: Discrete-time Control Systems. Prentice Hall, Englewood, Cliffs, USA, 1987.

[13] N.K. Bose: Properties of the Qn-matrix in bilinear transformation. Proc. IEEE, 71 (1983), 1110-1111.

[14] E.I. JURY: Inners and Stability of Dynamic Systems. Florida: Krieger, 1982, 242252.

[15] D. HENRION and J.B. LASSERRE: GloptiPoly: global optimization over polynomials with Matlab and SeDuMi. ACM Trans. on Mathematical Software, 29(2), (2003), 165-194.

[16] P. Boucher and D. Dumur: La Commande Prédictive. Collection Méthodes et pratiques de l'ingénieur, Editions Technip, Paris, 1996. 